\title{
Usefulness of virtual chromoendoscopy in the evaluation of subtle small bowel ulcerative lesions by endoscopists with no experience in videocapsule
}

Authors

Institutions
Mihai Rimbaş ${ }^{1,2}$, Denise Carmen Mihaela Zahiu' ${ }^{1}$ Andrei Mihai Voiosu ${ }^{1,2}$, Theodor Alexandru Voiosu ${ }^{1,2}$, Alina Ana-Maria Zlate ${ }^{1}$, Roxana Dinu ${ }^{1}$, Domenico Galasso ${ }^{3}$, Leonardo Minelli Grazioli ${ }^{3}$, Mariachiara Campanale ${ }^{3}$, Federico Barbaro ${ }^{3}$, Bogdan Radu Mateescu ${ }^{1,2}$, Bogdan Busuioc ${ }^{1}$, Tiberiu lordache ${ }^{1}$, Oana Dolofan ${ }^{1}$, Adelina Maria Popescu ${ }^{4}$, Vasile Daniel Balaban ${ }^{5}$, Mircea Mihai Raducan², Cristiano Spada ${ }^{3}$, Cristian Răsvan Băicuş², Guido Costamagna ${ }^{3}$

Institutions are listed at the end of article. submitted

2. December 2015

accepted after revision

29. March 2016

\section{Bibliography}

DOI http://dx.doi.org/

10.1055/s-0042-106206

Published online: 12.5.2016

Endoscopy International Open

2016; 04: E508-E514

(c) Georg Thieme Verlag KG

Stuttgart · New York

E-ISSN 2196-9736

\section{Corresponding author}

\section{Mihai Rimbaș MD, PhD}

Gastroenterology Department Colentina Clinical Hospital

19-21 Ştefan cel Mare Street 020125, Bucharest

Romania

Fax: +40-318162376 mrimbas@gmail.com

\section{License terms}

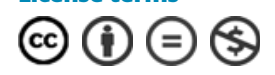

Background and study aims: In videocapsule endoscopy examination (VCE), subtle variations in mucosal hue or pattern such as those seen in ulcerations can be difficult to detect, depending on the experience of the reader. Our aim was to test whether virtual chromoendoscopy (VC) techniques, designed to enhance the contrast between the lesion and the normal mucosa, could improve the characterization of ulcerative mucosal lesions.

Patients and methods: Fifteen trainees or young gastroenterologists with no experience in VCE were randomly assigned to evaluate 250 true ulcerative and 100 false ulcerative, difficult-to-interpret small bowel lesions, initially as white light images (WLI) and then, in a second round, with the addition of one VC setting or again as WLI, labeling them as real lesions or artifacts.

\section{Introduction}

$\nabla$

Technological progress with videocapsule endoscopy (VCE) in the last 15 years has been impressive, making it an excellent technique for examining the small-bowel, with a diagnostic yield ranging from $55 \%$ to $100 \%$ [1-3], which helps guide proper management of patients in a significant proportion of cases [4]. However, it is not a perfect diagnostic tool and has a set of built-in limitations. The capsule cannot be steered or actively propelled, while the absence of bowel insufflation and the presence of residue or opaque fluid within the bowel lumen all interfere with the evaluation of the lesions, which are often visualized in less-than-perfect circumstances.

In order to overcome these limitations, videocapsule manufacturers have concentrated on devising ways to improve the quality of the captured data. One innovation is virtual chromoendoscopy (VC), a technique based on narrowing the bandwidth of the white light image [5], which at least
Results: On the overall image evaluation, an improvement in lesion characterization was observed by adding any chromoendoscopy setting, especially Blue mode and FICE 1, with increases in accuracy of $13 \%$ [95\%CI $0.8,25.3$ ] and $7.1 \%$ [95\%CI-17.0, 31.3], respectively. However, when only false ulcerative images were considered, with the same presets (Blue mode and FICE 1), there was a loss in accuracy of $10.7 \%$ [95\%CI$10.9,32.3]$ and $7.3 \%$ [95\%CI-1.3, 16.0], respectively. The interobserver agreement was poor for both readings.

Conclusions: VC helps beginner VCE readers correctly categorize difficult-to-interpret small bowel mucosal ulcerative lesions. However, false lesions tend to be misinterpreted as true ulcerative with the same presets. Therefore care is advised in using VC especially under poor bowel preparation.

theoretically facilitates characterization of subtle mucosal changes by increasing the contrast between adjacent mucosal areas. Recently, Flexible spectral Imaging Color Enhancement (FICE) technology and Blue mode, post-acquisition VC technologies have been incorporated into RAPID $^{\circledR}$ VCE reading software. Version 8 of RAPID ${ }^{\circledR}$ Reader provides 3 channels for FICE and 1 for Blue mode [6], allowing improved analysis of the mucosal patterns. This may represent an asset for beginner endoscopists as the enhanced contrast could improve their assessment of small-bowel mucosal lesions. However, data on virtual chromoendoscopy application in VCE are limited, yielding scanty and to a great extent discordant results, and even scarcer on experience in the hands of trainees [7-13].

The current study, therefore, was designed to test whether virtual chromoendoscopy settings available in a VCE reading system can help trainees or young gastroenterologists with no experience in VCE examination increase accuracy in correctly 

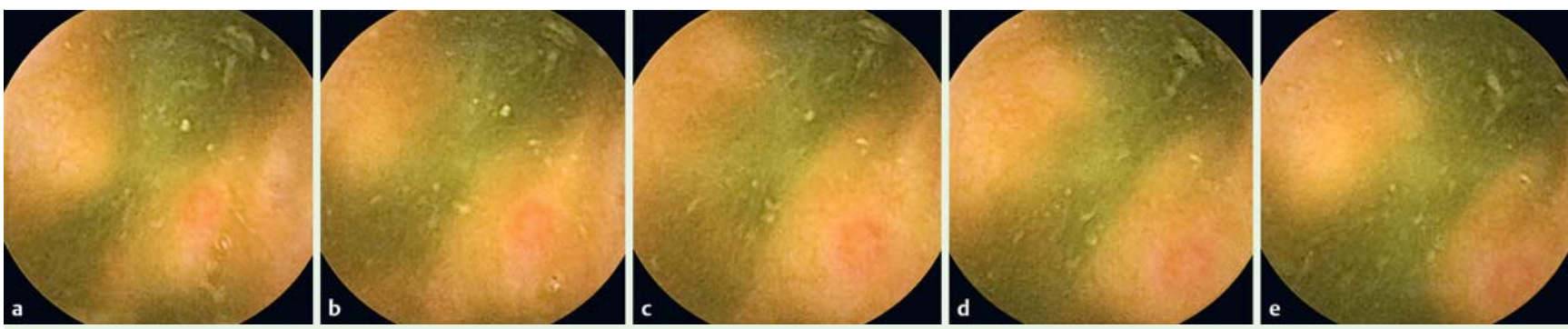

Fig. 1 Evaluation of a succession of frames depicting a small erosion (right lower quadrant) in the context of dirty luminal content; only the last image (e) was selected for the purpose of the study, being the least representative, but still suggestive for an ulcerative lesion.

categorizing difficult-to-interpret small bowel ulcerative lesions. We also assessed the contribution of each of the VC settings and tested interobserver reproducibility with and without VC.

\section{Patients and methods \\ $\nabla$}

This is a single-center, observational study based on a retrospective evaluation of $64 \mathrm{VCE}$ recordings performed in a tertiary care referral center as part of a clinical prospective study performed between October 2007 and December 2013, in which mild inflammatory involvement of the small bowel mucosa was anticipated in the majority of patients [14], either as part of their inflammatory systemic disease (they had a form of spondyloarthritis) or secondary to use of nonsteroidal anti-inflammatory drugs (NSAIDs). This report represents a different study from the one in which expert VCE readers tested the usefulness of virtual chromoendoscopy settings [15], in which some images from the current study were used and their evaluation was done following a different protocol.

\section{VCE procedure}

A PillCam ${ }^{\circledR}$ videocapsule (SB2 or SB2 L - the latter with 1 hour longer battery life-time, Given Imaging Co. Ltd., Yoqneam, Israel) was used in all cases following a standard examination protocol, with the PillCam administered after a patient had fasted for a minimum of 12 hours, ingested $3 \mathrm{~L}$ of polyethylene glycol (PEG)based bowel preparation (Endofalk; Dr. Falk Pharma GmbH, Freiburg, Germany), and swallowed an emulsion containing simethicone (Espumisan L; Berlin-Chemie AG, Berlin, Germany) just before initiation of the VCE examination. Details of the VCE procedure are also offered elsewhere [15].

\section{Selection of study images}

Careful review of all the VCE studies by a single, experienced reviewer (MR) using the RAPID $8^{\mathrm{TM}}$ software was performed to identify all mucosal defects that represented ulcerative lesions (defined as lesions with a pale or yellow base and a red or pink collar) [16] or artifacts that could mimic an ulcerative image. In that regard, an ulcerative image had to appear on 3 different images in the recording and to present a characteristic red halo to be considered true ulcerative. On the other hand, an artifact had to be clearly seen as an artifact (with no characteristic red halo, representing mainly white or yellow dirty luminal content) on at least 3 successive images. Any sequence of images for which there was doubt about whether the lesion was ulcerative or an artifact was abandoned and the evaluator proceeded to the next sequence of images. This process ended with selection of 2 sets of images: 250 true ulcerative (selected as the least representative visualization of an unequivocally confirmed ulcerative lesion from a succession of images, comprising small or shallow mucosal defects, erosions lacking a clear rim of erythema or located marginally in the field of view, or lesions with a poor image quality due to luminal content), and 100 false ulcerative (artifacts mimicking ulcerative lesions) ( $\bullet$ Fig. 1 and $\bullet$ Fig. 2). All these images were then mixed in a random order in a single set of 350 images, along with their chromoendoscopic correspondents (FICE 1,2 and 3, and Blue Mode).

This methodology also is described in detail elsewhere [15], the only difference being that the VCE studies were reviewed again using the same review process and in this study, the initial set of images was enriched with 50 more false ulcerative images in order to increase the statistical power of the false ulcerative set. The reference standard in image interpretation was considered their designation in the selection process (i.e., true ulcerative or not).

\section{Evaluation of the study images}

In a second phase, investigators from multiple centers with experience in gastrointestinal endoscopy were invited to evaluate the selected images. Fifteen trainees or young endoscopists with good experience in digestive endoscopy (with at least 1000 gastroscopies and 100 colonoscopies performed), but no experience in VCE examination, blinded to the original findings, analyzed the images in 2 steps. In the first round they evaluated the WLI images only, while in the second round, they were randomized in clusters of 3 to evaluate the same images with the addition of only 1 chromoendoscopy setting (FICE presets $1,2,3$ or Blue mode) or again in WLI. Each image had to be designated as either a true ulcerative lesion or as a false ulcerative one according to the investigator's opinion. The participants were not familiar with FICE and did not use it regularly in their practice, and prior to this study, they had not read a single VCE recording. Before reading the study images, they were given a short description of what an ulcerative lesion is and how it is defined (i.e. a lesion with a pale or yellow base and a red or pink collar), and were asked to translate that information from English into their native language (Romanian or Italian, respectively), in order to understanding for what they were about to search. The participants were encouraged to ask for further clarification before the images were presented to them. The 2 rounds of evaluation were performed by each of the readers at least 1 week apart.

\section{Statistical analysis}

The primary outcome measure of the study was the comparison of accuracies in correctly categorizing the lesions between the two evaluations (McNemar's stratified test). Calculation of the interobserver agreement value for each reading was also performed (Fleiss kappa; the $95 \%$ confidence interval was calculated 

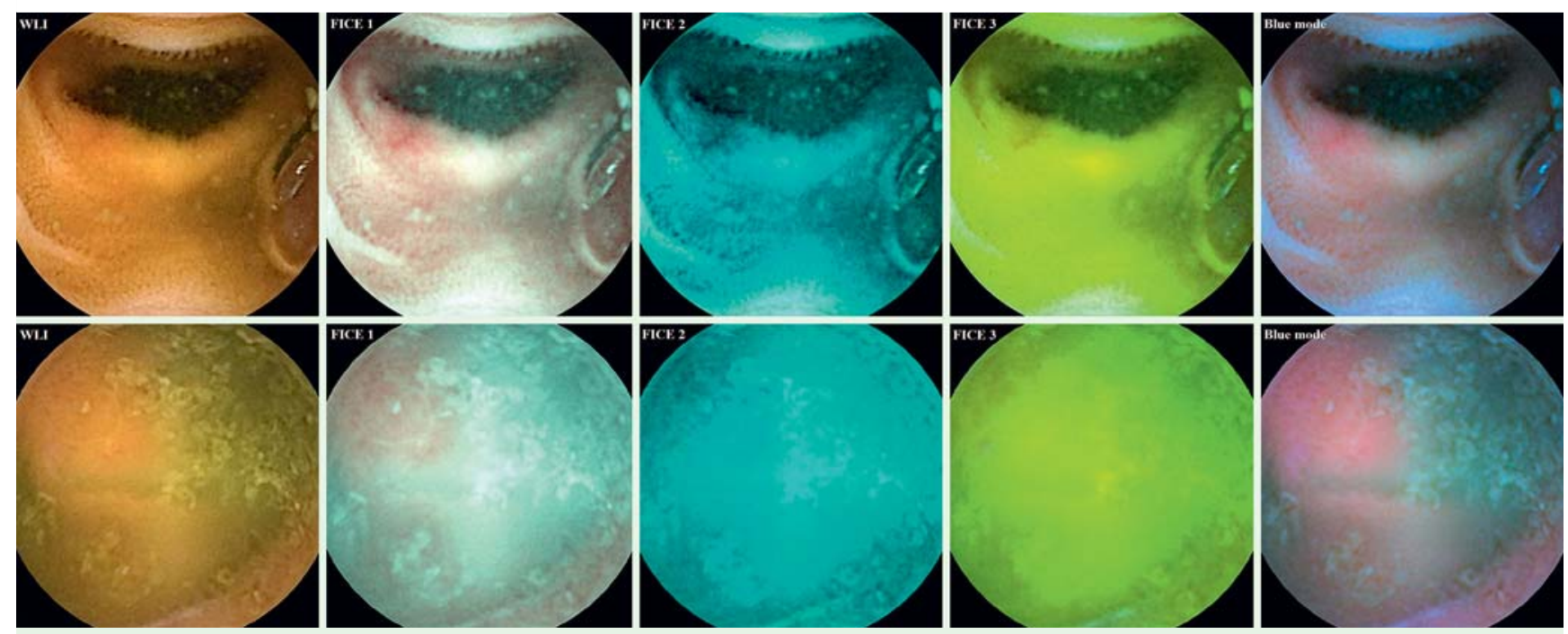

Fig.2 Images representing a true ulcerative lesion (upper set, left upper quadrant) and an artifact mimicking an ulcerative one (lower set, left upper quadrant) and their 4 chromoendoscopic correspondents.

by the bootstrap method). Statistical analyses were carried out with SPSS version 16.0 for Windows (SPSS Inc., Chicago, IL), Stata 11 (StataCorp, College Station, TX, USA), and WinPEPI 11.39 (JH Abramson, October 2013, http://www.brixtonhealth.com/). All statistical testing was two-sided.

\section{Results}

\section{$\nabla$}

\section{Overall image evaluation}

The results were very heterogeneous. When considering all 3 evaluations for each cluster of reviewers, there was a small decrease in accuracy for the second evaluation of WLI pictures (from 53.3 to $50.5 \%$ ) ( $\bullet$ Table 1 ). Adding any of the chromoendoscopy settings resulted in an improvement in correct characterization of a lesion as true or false ulcerative (for FICE from 56.5 to $63.7 \%$, for FICE 2 from 68.1 to $70.6 \%$, for FICE 3 from 57.8 to $62.7 \%$ and for Blue mode from 58.9 to $71.9 \%$ ), but statistical significance was obtained only for Blue mode ( $\bullet$ Table 1 and $\bullet$ Fig. 3 ).

\section{True ulcerative image evaluation}

Similar results were obtained when a subgroup analysis of the TRUE ulcerative image set was performed ( $\bullet$ Table 2$)$. Accuracy was decreased with WLI (from 43.3 to $37.1 \%$ ). For all VC settings, accuracy was increased (for FICE 1 from 44.9 to $57.8 \%$, for FICE 2 from 66.4 to $68.4 \%$, for FICE 3 from 49.7 to $55.7 \%$ and for Blue mode from 47.7 to $70.3 \%$ ). However, the results with stratified cluster evaluations were not statistically significant.

\section{False ulcerative image evaluation}

Regarding the FALSE ulcerative image set, there was a small gain in accuracy between the 2 rounds of evaluation for readers randomized to evaluate WLI images only (from 78.3 to $84.0 \%$ ). However, there was a loss of accuracy for VC presets that were most useful for TRUE image evaluation (for FICE 1 from 85.7 to $78.3 \%$ and for the Blue Mode from 86.7 to $76.0 \%$ ), while for the other 2, there was a small gain in accuracy (for FICE 2 from 72.3 to $76.0 \%$ and for FICE 3 from 78.0 to $80.0 \%$ ). Again, stratified evaluation resulted in no statistically significant differences ( $\square$ Table 3 ).

\section{Interobserver agreement}

The interobserver agreement was poor for both readings (WLI and chromoendoscopy-aided), with a maximum kappa value of 0.524 for the second evaluation of the study images by the investigators in the WLI group. A drop in interobserver agreement was noticed after adding any of the chromoendoscopy settings except for Blue mode ( Table 4$)$.

\section{Discussion}

$\nabla$

Our study shows that virtual chromoendoscopy (FICE and Blue mode technology) helps endoscopists with no experience in VCE interpretation better distinguish between true ulcerative lesions and artifacts that mimic ulcerations. The increment in accuracy was $13.0 \%$ for Blue mode and $7.1 \%$ for FICE 1 in a set of 350 selected difficult-to-interpret, potentially ulcerative small bowel lesions. However, statistical significance was obtained only for Blue mode.

In our view, the potential of VC could be underestimated if only clearly visible mucosal lesions were to be evaluated. The true added benefit of VC over WLI should be tested in less clearly visualized pathologic changes of the mucosa because many ulcerative lesions with poor contrast are difficult to characterize precisely with conventional imaging [8]. Because almost no randomized studies in this regard have been performed to date, it seemed important to design methods to certify the degree of improvement in the detectability of such small bowel lesions, presuming that chromoendoscopy-aided VCE might help better characterize arguable erosions or ulcerations that are less clearly visualized in WLI.

Herein we use a novel concept of intestinal lesion in the clinical setting of inflammatory mucosal involvement. The lesions evaluated in the current study were intentionally made more difficult to detect and interpret, a situation that may be encountered frequently in VCE examinations. They were carefully selected to be either small, located marginally or distant in the field of view, seen tangentially or visualized under poor visibility because of the luminal presence of air bubbles, food residue, and bile pigment. 
Table 1 Performance characteristics in the OVERALL evaluation of the study images.

\begin{tabular}{|c|c|c|c|c|}
\hline Randomized VC setting & Evaluator & Accuracy for the first reading & Accuracy for the second reading & Difference in accuracy $(\%)[95 \% \mathrm{Cl}]$ \\
\hline \multirow[t]{4}{*}{ WLI } & 1 & $61.7[56.5,66.7]$ & $54.3[49.0,59.4]$ & $-7.4[-12.5,-2.3]^{1}$ \\
\hline & 2 & $49.1[43.9,54.4]$ & $44.6[39.5,49.8]$ & $-4.6[-8.8,-0.3]^{1}$ \\
\hline & 3 & $49.1[43.9,54.4]$ & $52.6[47.3,57.7]$ & $3.4[0.0,6.8]^{1}$ \\
\hline & Globally ${ }^{2}$ & 53.3 & 50.5 & $-2.9[-9.2,3.5]$ \\
\hline \multirow[t]{4}{*}{ FICE 1} & 4 & $63.4[58.3,68.3]$ & $52.3[47.1,57.5]$ & $-11.1[-16.2,-6.0]^{1}$ \\
\hline & 5 & $61.4[56.2,66.4]$ & $63.4[58.3,68.3]$ & $2.0[-3.3,7.3]$ \\
\hline & 6 & $44.7[39.6,49.9]$ & $75.4[70.6,79.6]$ & $30.7[24.3,36.7]^{1}$ \\
\hline & Globally ${ }^{2}$ & 56.5 & 63.7 & $7.1[-17.0,31.3]$ \\
\hline \multirow[t]{4}{*}{ FICE 2} & 7 & $63.1[58.0,68.0]$ & $73.4[68.6,77.8]$ & $10.3[5.3,15.2]^{1}$ \\
\hline & 8 & $65.4[60.3,70.2]$ & $58.9[53.6,63.9]$ & $-6.6[-12.0,-1.1]^{1}$ \\
\hline & 9 & $75.7[71.0,79.9]$ & $79.4[74.9,83.3]$ & $3.7[-0.5,7.9]$ \\
\hline & Globally² & 68.1 & 70.6 & $2.5[-7.1,12.1]$ \\
\hline \multirow[t]{4}{*}{ FICE 3} & 10 & $62.0[56.8,66.9$ & $77.7[73.1,81.8]$ & $15.7[10.1,21.2]^{1}$ \\
\hline & 11 & $58.9[53.6,63.9]$ & $50.6[45.4,55.8]$ & $-8.3[-14.2,-2.2]^{1}$ \\
\hline & 12 & $52.6[47.3,57.7]$ & $59.7[54.5,64.7]$ & $7.1[1.3,12.9]^{1}$ \\
\hline & Globally² & 57.8 & 62.7 & $4.9[-8.9,18.6]$ \\
\hline \multirow[t]{4}{*}{ Blue Mode } & 13 & $36.9[32.0,42.0]$ & $62.3[57.1,67.2]$ & $25.4[19.1,31.4]^{1}$ \\
\hline & 14 & $71.7[66.8,76.2]$ & $77.1[72.5,81.2]$ & $5.4[1.0,9.8]^{1}$ \\
\hline & 15 & $68.0[62.9,72.7]$ & $76.3[71.6,80.4]$ & $8.3[3.3,13.3]^{1}$ \\
\hline & Globally² & 58.9 & 71.9 & $13.0[0.8,25.3]^{1}$ \\
\hline
\end{tabular}

VC, virtual chromoendoscopy; WLI, white light image; CI, confidence interval

${ }^{1}$ statistically significant

2 stratified McNemar test

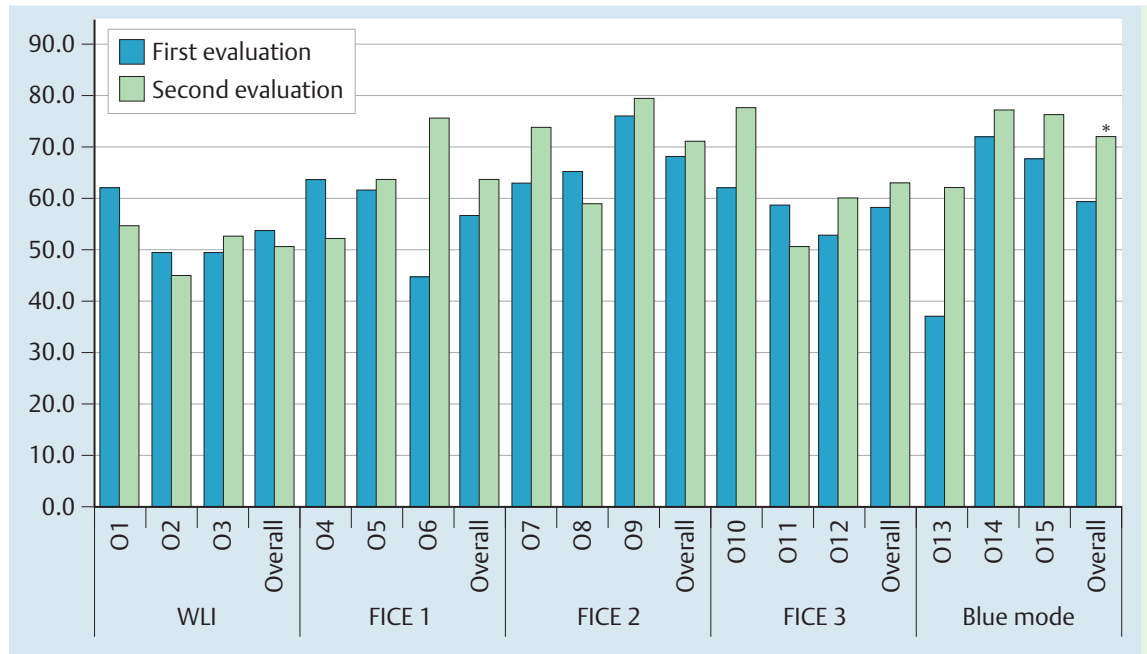

Fig. 3 Comparison of accuracy in correctly identifying the ulcerative images for all readers at their first and second evaluations. The investigators were randomized for the second reading to evaluate the study images in $\mathrm{WLI}(\mathrm{O} 1-3)$, FICE $1(\mathrm{O} 4-6)$, FICE $2(07-9)$, FICE $3(010-12)$ and Blue mode (013-15). Of the overall evaluations, statistical significance was obtained only for Blue mode $\left({ }^{*}\right)$; WLI, white light imaging; O, observer.

We chose an intuitive approach to videocapsule reading. First the examiners evaluated the recordings in WLI, then they assessed whether VCE settings helped them better perceive the characteristic appearance of an ulcerative lesion. In real-life practice, the tendency is to try all chromoendoscopy settings (FICE presets 1, 2 and 3 and Blue mode) when analyzing doubtful images, however, in our study, we wanted to investigate the contribution of each of the VC settings. Our results indicate that Blue mode and possibly FICE 1 may be the preferred settings for beginner reviewers because they significantly improve characterization of difficult-to -interpret ulcerative lesions.

Up to now, VCE's ability to visualize ulcerative intestinal lesions has been useful for studying the adverse effects of NSAIDs on the small bowel mucosa [17] and for evaluation of patients with known or suspected small bowel Crohn's disease [2-4]. Recently however, focus has shifted to performance of VC-aided VCE in identification of ulcerative lesions of the small bowel. In the first published case report on this issue [18], FICE imaging was considered to be superior to WLI evaluation of the surface mucosal characteristics in a patient with mucosal inflammation and ulceration compatible with active small bowel Crohn's disease. Contrary to this report, 2 small studies (with a maximum of 44 ulcerative lesions evaluated) did not find statistically significant differences between conventional WLI-VCE and VCE-FICE $[12,19]$ in detection of erosions or ulcerations, although in one of them, the addition of FICE improved detection of ulcerative lesions (from 32 up to 44 lesions, $27.3 \%$ improvement) [12]. Other investigators reported improved quality of images of erosions/ulcerations when FICE was used (up to $53.3 \%$ image improvement for the evaluation of 47 erosions and ulcerations) [10,20]. Furthermore, experience with Blue mode application in SB-VCE reading is even more limited $[11,21]$; it was found to offer image improvement in $93 \%$ of 60 ulcers and aftae in only one study involving VCE recordings from 52 patients [8]. 
Table 2 Performance characteristics in the evaluation of the TRUE ULCERATIVE image set.

\begin{tabular}{|c|c|c|c|c|}
\hline Randomized VC setting & Evaluator & Accuracy for the first reading & Accuracy for the second reading & Difference in accuracy (\%) [95\% Cl] \\
\hline \multirow[t]{4}{*}{ WLI } & 1 & $56.4[50.2,62.4]$ & $44.0[38.0,50.2]$ & $-12.4[-18.4,-6.2]^{1}$ \\
\hline & 2 & $34.8[29.2,40.9]$ & $29.2[23.9,35.1]$ & $-5.6[-11.0,-0.2]^{1}$ \\
\hline & 3 & $38.8[33.0,45.0]$ & $38.0[32.2,44.2]$ & $-0.8[-4.4,2.8]$ \\
\hline & Globally ${ }^{2}$ & 43.3 & 37.1 & $-6.3[-12.9,0.3]$ \\
\hline \multirow[t]{4}{*}{ FICE 1} & 4 & $49.6[43.5,55.8]$ & $33.6[28.0,39.7]$ & $-16.0[-22.8,-9.0]^{1}$ \\
\hline & 5 & $52.8[46.6,58.9]$ & $59.2[53.0,65.1]$ & $6.4[0.0,12.7]^{1}$ \\
\hline & 6 & $32.1[26.6,38.2]$ & $80.7[75.4,85.1]$ & $48.6[41.4,54.9]^{1}$ \\
\hline & Globally² & 44.9 & 57.8 & $12.9[-24.1,50.0]$ \\
\hline \multirow[t]{4}{*}{ FICE 2} & 7 & $54.4[48.2,60.5]$ & $69.2[63.2,74.6]$ & $14.8[8.7,20.7]^{1}$ \\
\hline & 8 & $54.8[48.6,60.9]$ & $44.4[38.4,50.6]$ & $-10.4[-17.5,-3.1]^{1}$ \\
\hline & 9 & $90.0[85.7,93.1]$ & $91.6[87.5,94.4]$ & $1.6[-2.6,5.9]$ \\
\hline & Globally² & 66.4 & 68.4 & $2.0[-12.3,16.3]$ \\
\hline \multirow[t]{4}{*}{ FICE 3} & 10 & $53.2[47.0,59.3]$ & $79.2[73.7,83.8]$ & $26.0[19.4,32.2]^{1}$ \\
\hline & 11 & $51.2[45.0,57.3]$ & $41.6[35.7,47.8]$ & $-9.6[-16.7,-2.4]^{1}$ \\
\hline & 12 & $44.8[38.8,51.0]$ & $46.4[40.3,52.6]$ & $1.6[-5.5,8.7]$ \\
\hline & Globally² & 49.7 & 55.7 & $6.0[-14.6,26.6]$ \\
\hline \multirow[t]{4}{*}{ Blue Mode } & 13 & $12.8[9.2,17.5]$ & $58.4[52.2,64.3]$ & $45.6[39.0,51.6]^{1}$ \\
\hline & 14 & $62.4[56.3,68.2]$ & $77.2[71.6,82.0]$ & $14.8[9.9,19.7]^{1}$ \\
\hline & 15 & $68.0[62.0,73.5]$ & $75.2[69.5,80.1]$ & $7.2[1.5,12.8]^{1}$ \\
\hline & Globally² & 47.7 & 70.3 & $22.5[-0.5,45.5]^{3}$ \\
\hline
\end{tabular}

VC, virtual chromoendoscopy; WLI, white light image; Cl, confidence interval

1 statistically significant

2 stratified McNemar test

${ }^{3} \mathrm{p}=0.07$

Table4 Interobserver agreement in the evaluation of the study images.

\begin{tabular}{|l|ll} 
Randomization & $\begin{array}{l}\text { Kappa value with 95\% } \\
\text { confidence intervals for } \\
\text { the first evaluation } \\
\text { (WLI only) }\end{array}$ & $\begin{array}{l}\text { Kappa value with 95\% } \\
\text { confidence intervals for } \\
\text { the second evaluation } \\
\text { (VC-aided) }\end{array}$ \\
\hline WLI & $0.395[0.382-0.415]^{1}$ & $0.524[0.477-0.565]$ \\
\hline FICE 1 & $0.317[0.267-0.384]$ & $0.097[0.040-0.151]$ \\
\hline FICE 2 & $0.150[0.122-0.195]$ & $0.092[0.086-0.119]$ \\
\hline FICE 3 & $0.344[0.329-0.383]$ & $0.235[0.191-0.267]$ \\
\hline Blue Mode & $0.205[0.158-0.272]$ & $0.302[0.280-0.380]$ \\
\hline
\end{tabular}

WLI, white light endoscopic imaging; VC, virtual chromoendoscopy

1 For all 15 evaluators, for the initial WLI reading, the kappa value was 0.306 [0.293-0.321]

The scarcity and contradictory character of the results reported so far, compounded with the varying methodologies and small sample sizes, bring into question the true impact of this novel adjunct technology in the practice of VCE examination. Moreover, because VCE trainees are predisposed to miss small erosions/ulcerations [22,23], the main aim of our study was to evaluate the diagnostic accuracy of VC in VCE and to determine if it provides additional value over conventional examination in the hands of beginner examiners. To our knowledge, the only published studies to date involving trainees show that the detectability of erosions/ulcerations obscured by the opaque luminal content was improved (by 30\%) using VCE-FICE [23], and that sensitivity and specificity was insignificantly increased when adding FICE for evaluation of lesions with high bleeding potential [19]. Both studies are affected by the inclusion of relatively small sets of images (82 and 27 ulcerative lesions, respectively) and the involvement of very few examiners, which makes ours the largest study to date on this issue. Besides randomization of the VC evaluations (including a group evaluating in the second round the same WLI images without the aid of VC), the merit of our metho- dology exists also in the fact that all of the selected pathologic images, despite being difficult to interpret, were still suggestive of an ulcerative lesion in WLI ( $\bullet$ Fig. 1 ), and that they had an artifact comparison.

Few clinical studies to date have examined which chromoendoscopy settings perform better in specific circumstances. Pohl et al. [18] and Gupta et al. [19] assumed in their works that FICE preset 1 achieved the preferred appearance, while Duque et al. [10] felt that FICE preset 2 was most useful to better increase the contrast between the pathologic areas and the background mucosa. However, this approach seems biased as the groups used only these preferred settings in their studies and did not adequately compare all VC presets. Three independent studies $[8,20,23]$, including 1 involving VCE trainees, and our previous study involving experts [15] concluded that VCE readers significantly improve their detectability of erosions/ulcerations using FICE presets 1 and 2, but not preset 3. Still, in another study performed by Krystallis et al. [11] involving 60 ulcers and aftae (the only one in which WLI, FICE, and Blue filter mode all were evaluated), Blue filter mode provided image improvement (compared to WLI) in $93 \%$, with improvement being observed in only $36.6 \%, 3 \%$ and $3 \%$ for FICE presets 1, 2 and 3, respectively.

In fact, at present there are no recommendations regarding which setting(s) to use when examining a potential mucosal lesion so as to better discern or characterize it, and every reader is choosing settings as a matter of personal preference. We proved in our studies that VC is useful for better characterizing difficultto-interpret ulcerative lesions. Therefore, our proposed strategy for less experienced VCE readers would be to first evaluate the recordings in WLI, and then to use VC settings Blue mode and FICE 1 to better define or characterize an arguable ulcerative lesion. However, readers should be advised that the same presets misguide classification of artifacts as false ulcerative lesions. Therefore, care is needed in the context of dirty luminal content. Like us, other investigators have found that in poor bowel visibility 
Table 3 Performance characteristics in the evaluation of the FALSE ULCERATIVE image set.

\begin{tabular}{|c|c|c|c|c|}
\hline Randomized VC setting & Evaluator & Accuracy for the first reading & Accuracy for the second reading & Difference in accuracy (\%) [95\% Cl] \\
\hline \multirow[t]{4}{*}{ WLI } & 1 & $75[65.7,82.5]$ & $80[71.1,86.7]$ & $5[-3.8,13.8]$ \\
\hline & 2 & $85[76.7,90.7]$ & $83[74.5,89.1]$ & $-2[-8.9,4.8]$ \\
\hline & 3 & $75[65.7,82.5]$ & $89[81.4,93.7]$ & $14[6.4,22.1]^{1}$ \\
\hline & Globally² & 78.3 & 84.0 & $5.7[-3.4,14.7]$ \\
\hline \multirow[t]{4}{*}{ FICE 1} & 4 & $98[93.0,99.4]$ & $99[94.6,99.8]$ & $1[-3.7,6.1]$ \\
\hline & 5 & $83[74.5,89.1]$ & $74[64.6,81.6]$ & $-9[-18.5,5.0]$ \\
\hline & 6 & $76[66.8,83.3]$ & $62[52.2,70.9]$ & $-14[-23.2,-14.5]^{1}$ \\
\hline & Globally² & 85.7 & 78.3 & $-7.3[-16.0,1.3]$ \\
\hline \multirow[t]{4}{*}{ FICE 2} & 7 & $85[76.7,90.7]$ & $84[75.6,89.9]$ & $-1[-10.0,8.0]$ \\
\hline & 8 & $92[85.0,95.9]$ & $95[88.8,97.8]$ & $3[-3.7,10.0]$ \\
\hline & 9 & $40[30.9,49.8]$ & $49[39.4,58.7]$ & $9[-1.6,19.2]$ \\
\hline & Globally² & 72.3 & 76.0 & $3.7[-2.0,9.4]$ \\
\hline \multirow[t]{4}{*}{ FICE 3} & 10 & $84[75.6,89.9]$ & $74[64.6,81.6]$ & $-10[-19.3,-7.0]^{1}$ \\
\hline & 11 & $78[68.9,85.0]$ & $73[63.6,80.7]$ & $-5[-15.9,6.1]$ \\
\hline & 12 & $72[62.5,79.9]$ & $93[86.3,96.6]$ & $21[10.9,31.0]^{1}$ \\
\hline & Globally² & 78.0 & 80.0 & $2.0[-16.8,20.8]$ \\
\hline \multirow[t]{4}{*}{ Blue Mode } & 13 & $97[91.5,99.0]$ & $72[62.5,79.9]$ & $-25[-34.5,-15.8]^{1}$ \\
\hline & 14 & $95[88.8,97.8]$ & $77[67.8,84.2]$ & $-18[-26.5,-10.4]^{1}$ \\
\hline & 15 & $68[58.3,76.3]$ & $79[70.0,85.8]$ & $11[4.0,21.3]^{1}$ \\
\hline & Globally² & 86.7 & 76.0 & $-10.7[-32.3,10.9]$ \\
\hline
\end{tabular}

VC, virtual chromoendoscopy; WLI, white light image; $\mathrm{Cl}$, confidence interval

${ }^{1}$ statistically significant

2 stratified McNemar test

conditions, the frequency of false-positive ulcerative findings was increased with VCE-FICE, as compared to conventional WLI $[19,23]$. This proves once more that there is a major limitation when using VC without first reviewing the VCE recording with WLI, because electronic processing denaturizes the true colors of the image.

A secondary aim of our study was to determine if outcomes of interpretation of VCE-FICE images by inexperienced examiners were reproducible. In consequence, a surprising finding is that the measure of agreement, though poor, decreases after adding chromoendoscopy. This would seem to imply that lesions' characterization becomes less reproducible with the addition of the new technology, because alteration of image characteristics results in its more unpredictable interpretation.

The potential limitations of our study are that the investigators reviewed static images and we did not know the percentage of real and false ulcerative images encountered in VCE studies (which probably varies from indication to indication and patient to patient). Moreover, despite use of a very rigorous methodology to ensure what every image represented, the selection process was subjective, as was classification of every separate image as "difficult to interpret" and thus included in the study database. Last, whether the observed differences in accuracy translate into a clinical benefit for the patients remains unknown, particularly considering that the producers are now offering a better version of PillCam (SB3) with adaptive frame rate, which will create more images for evaluation, and thus provide improved image quality. In conclusion, virtual chromoendoscopy (especially Blue filter mode) helps beginner VCE readers to correctly categorize small bowel mucosal ulcerative lesions. However, false lesions also tend to be misinterpreted as true ulcerative, especially with the above preset. Therefore care must be taken in using VCE especially when bowel preparation is poor. Moreover, this proof-of-concept study shows that there is potential for improvement in this application in small bowel endoscopy, but a better understanding of and refinements in VC technology are definitely required in order to further improve characterization of small bowel lesions.

Competing interests: Cristiano Spada and Guido Costamagna were consultants for Given Imaging, Ltd.

\section{Institutions}

${ }^{1}$ Gastroenterology Department, Colentina Clinical Hospital, Bucharest, Romania

2 Internal Medicine Department, Carol Davila University of Medicine,

Bucharest, Romania

${ }^{3}$ Digestive Endoscopy Unit, Catholic University, Rome, Italy

${ }^{4}$ Gastroenterology Department, University Emergency Hospital, Bucharest, Romania

${ }^{5}$ Gastroenterology Department, Emergency Military Hospital, Bucharest, Romania

\section{Acknowledgements}

\section{$\nabla$}

This work has been funded by the Sectoral Operational Programme Human Resources Development 2007-2013 of the Ministry of the European Funds through the Financial Agreement POSDRU/159/1.5/S/132395.

\section{References}

1 Rastogi A, Schoen RE, Slivka A. Diagnostic yield and clinical outcomes of capsule endoscopy. Gastrointest Endosc 2004; 60: 959-964

2 Solem CA, Loftus EV, Fletcher JG et al. Small-bowel imaging in Crohn's disease: a prospective, blinded, 4-way comparison trial. Gastrointest Endosc 2008; 68: 255-266

3 Jensen MD, Nathan T, Rafaelsen SR et al. Diagnostic accuracy of capsule endoscopy for small bowel Crohn's disease is superior to that of MR enterography or CT enterography. Clin Gastroenterol Hepatol 2011; 9: $124-129$

4 Pennazio M, Spada C, Eliakim R et al. Small-bowel capsule endoscopy and device-assisted enteroscopy for diagnosis and treatment of smallbowel disorders: European Society of Gastrointestinal Endoscopy (ESGE) Clinical Guideline. Endoscopy 2015; 47: 352 - 376

5 Trivedi PJ, Braden B. Indications, stains and techniques in chromoendoscopy. QJM 2013; 106: 117-131 
6 Pohl J, Aschmoneit I, Schumann $S$ et al. Computed image modification for enhancement of small-bowel surface structures at video capsule endoscopy. Endoscopy 2010; 42: 490-492

7 Osawa H, Yamamoto H. Present and future status of flexible spectral imaging color enhancement and blue laser imaging technology. Dig Endosc 2014; 26: 105-115

8 Konishi M, Shibuya T, Mori $\mathrm{H}$ et al. Usefulness of flexible spectral imaging color enhancement for the detection and diagnosis of small intestinal lesions found by capsule endoscopy. Scand J Gastroenterol 2014; 49: $501-505$

9 Kobayashi $Y$, Watabe H, Yamada A et al. Efficacy of flexible spectral imaging color enhancement on the detection of small intestinal diseases by capsule endoscopy. J Dig Dis 2012; 13: 614-620

10 Duque G, Almeida N, Figueiredo P et al. Virtual chromoendoscopy can be a useful software tool in capsule endoscopy. Rev Esp Enferm Dig 2012; 104: 231-236

11 Krystallis C, Koulaouzidis A, Douglas $S$ et al. Chromoendoscopy in small bowel capsule endoscopy: Blue mode or Fuji Intelligent Colour Enhancement? Dig Liver Dis 2011; 43: 953-957

12 Imagawa H, Oka S, Tanaka S et al. Improved detectability of small-bowel lesions via capsule endoscopy with computed virtual chromoendoscopy: a pilot study. Scand J Gastroenterol 2011; 46: 1133-1137

13 Cotter J, Magalhães J, Castro FD et al. Virtual chromoendoscopy in small bowel capsule endoscopy: new light or a cast of shadow? World J Gastrointest Endosc 2014; 6: 359-365

14 Rimbaș M, Marinescu M, Voiosu MR et al. NSAID-induced deleterious effects on the proximal and mid small bowel in seronegative spondyloarthropathy patients. World J Gastroenterol 2011; 17: 1030 - 1035

15 Rimbas M, Negreanu L, Ciobanu $L$ et al. Is virtual chromoendoscopy useful in the evaluation of subtle ulcerative small bowel lesions detect- ed by videocapsule endoscopy? Endoscopy International Open 2015; 3: E615-E620

16 Gralnek IM, Defranchis R, Seidman E et al. Development of a capsule endoscopy scoring index for small bowel mucosal inflammatory change. Aliment Pharmacol Ther 2008; 27: 146 - 154

17 Goldstein JL, Eisen GM, Lewis B et al. Small bowel mucosal injury is reduced in healthy subjects treated with celecoxib compared with ibuprofen plus omeprazole, as assessed by video capsule endoscopy. Aliment Pharmacol Ther 2007; 25: 1211 -1222

18 Pohl J, Aschmoneit I, Schumann S et al. Computed image modification for enhancement of small-bowel surface structures at video capsule endoscopy. Endoscopy 2010; 42: 490-492

19 Gupta T, Ibrahim M, Deviere J et al. Evaluation of Fujinon intelligent chromo endoscopy-assisted capsule endoscopy in patients with obscure gastroenterology bleeding. World J Gastroenterol 2011; 17: $4590-4595$

20 Imagawa H, Oka S, Tanaka S et al. Improved visibility of lesions of the small intestine via capsule endoscopy with computed virtual chromoendoscopy. Gastrointestinal Endoscopy 2011; 73: 299-306

21 Koulaouzidis A, Douglas S, Plevris JN. Blue mode does not offer any benefit over white light when calculating Lewis score in small-bowel capsule endoscopy. World J Gastrointest Endosc 2012; 4: 33-37

22 DeLeusse A, Landi B, Edery J et al. Video capsule endoscopy for investigation of obscure gastrointestinal bleeding: feasibility, results, and interobserver agreement. Endoscopy 2005; 37: 617-621

23 Sakai E, Endo H, Kato $S$ et al. Capsule endoscopy with flexible spectral imaging color enhancement reduces the bile pigment effect and improves the detectability of small bowel lesions. BMC Gastroenterol 2012; 12: 83 